Western University

Scholarship@Western

Oncology Publications

Oncology Department

$1-2009$

\title{
Adaptive radiation therapy for localized mesothelioma with mediastinal metastasis using helical tomotherapy.
}

James Renaud

Slav Yartsev

A Rashid Dar

Jacob Van Dyk

The University of Western Ontario, vandyk@uwo.ca

Follow this and additional works at: https://ir.lib.uwo.ca/oncpub

Part of the Medical Biophysics Commons, and the Oncology Commons

Citation of this paper:

Renaud, James; Yartsev, Slav; Dar, A Rashid; and Van Dyk, Jacob, "Adaptive radiation therapy for localized mesothelioma with mediastinal metastasis using helical tomotherapy." (2009). Oncology Publications. 127.

https://ir.lib.uwo.ca/oncpub/127 


\section{Adaptive Radiation Therapy for Localized Mesothelioma with Mediastinal Metastasis Using Helical Tomotherapy}

Article in Medical dosimetry: official journal of the American Association of Medical Dosimetrists · February 2009 DOI: 10.1016/j.meddos.2008.10.002 · Source: PubMed

CITATIONS

5

4 authors, including:

James Renaud

McGill University

20 PUBLICATIONS 17 CITATIONS

SEE PROFILE
READS

99

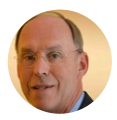

Jacob (Jake) Van Dyk

Western University

234 PUBLICATIONS 5,297 CITATIONS

SEE PROFILE

Some of the authors of this publication are also working on these related projects: 
Provided for non-commercial research and education use. Not for reproduction, distribution or commercial use.
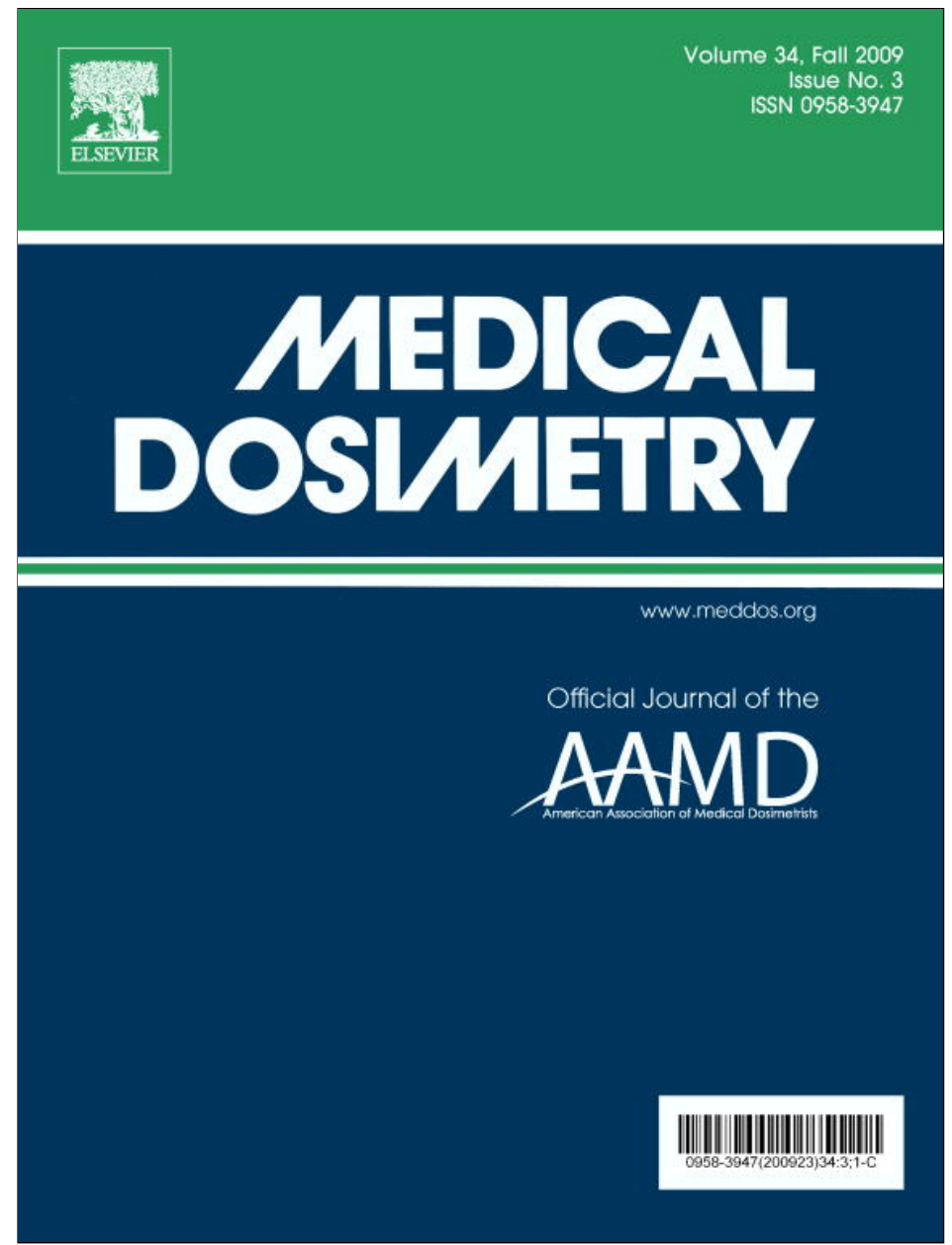

This article appeared in a journal published by Elsevier. The attached copy is furnished to the author for internal non-commercial research and education use, including for instruction at the authors institution and sharing with colleagues.

Other uses, including reproduction and distribution, or selling or licensing copies, or posting to personal, institutional or third party websites are prohibited.

In most cases authors are permitted to post their version of the article (e.g. in Word or Tex form) to their personal website or institutional repository. Authors requiring further information regarding Elsevier's archiving and manuscript policies are encouraged to visit:

http://www.elsevier.com/copyright 


\title{
ADAPTIVE RADIATION THERAPY FOR LOCALIZED MESOTHELIOMA WITH MEDIASTINAL METASTASIS USING HELICAL TOMOTHERAPY
}

\author{
James Renaud, Slav Yartsev, Ph.D., A. Rashid Dar, M.D., F.R.C.P.C., and \\ JAKE VAN DYK, M.SC., F.C.C.P.M.
}

Departments of Physics and Engineering and Radiation Oncology, London Regional Cancer Program, London Health Sciences Centre, London, Ontario, Canada; and Department of Oncology, The University of Western Ontario, London, Ontario, Canada

(Received 2 July 2008; accepted 23 October 2008)

\begin{abstract}
The purpose of this study was to compare 2 adaptive radiotherapy strategies with helical tomotherapy. A patient having mesothelioma with mediastinal nodes was treated using helical tomotherapy with pretreatment megavoltage CT (MVCT) imaging. Gross tumor volumes (GTVs) were outlined on every MVCT study. Two alternatives for adapting the treatment were investigated: (1) keeping the prescribed dose to the targets while reducing the dose to the OARs and (2) escalating the target dose while maintaining the original level of healthy tissue sparing. Intensity modulated radiotherapy (step-and-shoot IMRT) and 3D conformal radiotherapy (3DCRT) plans for the patient were generated and compared. The primary lesion and nodal mass regressed by $16.2 \%$ and $32.5 \%$, respectively. Adapted GTVs and reduced planning target volume (PTV) margins of $4 \mathrm{~mm}$ after 22 fractions decrease the planned mean lung dose by $19.4 \%$. For dose escalation, the planned prescribed doses may be increased from 50.0 to $58.7 \mathrm{~Gy}$ in $\mathrm{PTV}_{1}$ and from 60.0 to $70.5 \mathrm{~Gy}$ in $\mathrm{PTV}_{2}$. The step-and-shoot IMRT plan was better in sparing healthy tissue but did not provide target coverage as well as the helical tomotherapy plan. The 3DCRT plan resulted in a prohibitively high planned dose to the spinal cord. MVCT studies provide information both for setup correction and plan adaptation. Improved healthy tissue sparing and/or dose escalation can be achieved by adaptive planning. (c) 2009 American Association of Medical Dosimetrists.
\end{abstract}

Key Words: Helical tomotherapy, Mesothelioma, Adaptive radiotherapy.

\section{INTRODUCTION}

Malignant mesothelioma is a very serious disease. The contemporary treatment in operable cases involves a combination of neoadjuvant chemotherapy (Cisplatinum, Premetrex), extrapleural pneumonectomy, and postoperative radical radiation therapy. In these patients, newer intensity modulated radiation therapy (IMRT) techniques are used to achieve a better therapeutic ratio. ${ }^{1,2}$ However, the majority of cases (70-80\%) are inoperable and often require palliative radiation therapy. ${ }^{3}$ Clinical use of helical tomotherapy (HT) has not been reported in these situations.

Escalating the prescribed radiation dose to the planning target volume (PTV) improves the likelihood of tumor control and long-term survival, but is limited by the tolerance doses of nearby organs at risk (OARs)., ${ }^{4,5}$ To raise the tumor dose without increasing the risk of normal-tissue toxicity, precise delivery and verification systems such as step-and-shoot IMRT, CyberKnife, and HT have been developed. ${ }^{6-10}$

HT is composed of a 6-MeV linear accelerator mounted on a ring gantry, with radiation delivered to the patient in a helical fashion. A 64-leaf collimator allows

Reprint requests to: Slav Yartsev, Ph.D., LRCP/LHSC, 790 Commissioners Road, East, London, Ontario N6A 4L6, Canada. E-mail: slav.yartsev@1hsc.on.ca for a dynamically adjustable intensity profile. ${ }^{11,12}$ In addition to delivering highly conformal radiation dose distributions, HT is equipped with xenon detectors designed to obtain megavoltage computed tomography (MVCT) image sets of the patient prior to treatment. ${ }^{13}$ Radiation therapists co-register these MVCT studies with planning kilovoltage $\mathrm{CT}$ ( $\mathrm{kVCT}$ ) studies to correctly position the patient, thereby minimizing setup error. Pretreatment MVCT imaging provides enough soft tissue contrast to reliably delineate organs and lesions, enabling clinicians to monitor interfraction variations in patient anatomy. The quantification of tumor regression occurring during radiotherapy in lung cancer patients is well documented. ${ }^{4,14-17}$ Varying degrees of shrinkage have been observed, but in all cases, as a tumor decreases in volume, healthy tissue that takes its place is unnecessarily irradiated to the prescription dose. Plan adaptation to these anatomy changes may significantly improve a patient's treatment. ${ }^{18}$ Woodford et al. have related the potential benefit of image-guided adaptive radiotherapy to changes in gross tumor volume (GTV). They found that regression patterns could be broadly divided into 3 groups and that adaptive planning would be most appropriate when the GTV decreases by greater than $30 \%$ at any point in the first 20 fractions of treatment. ${ }^{17}$

In some cases, tumors exhibit resistance to treatment, and no clear trend toward a decrease in volume is 
observed. This reduced radiosensitivity in some tumor cells may be the result of several factors, one of which is the 'hypoxia effect.' 19 Tumors have deficient vascular systems and as such, contain hypoxic cells, which survive in nutrient-deprived and low oxygen conditions and have a characteristically low sensitivity to radiation levels. Their presence significantly hinders GTV regression and negatively influences prognosis. ${ }^{19}$

Because of potentially delayed responses, adaptive radiotherapy may offer little benefit to these types of resistive tumors. If, however, the margins defining the PTV are reduced, a new and improved plan may be created. PTV margins encompass intrafractional motion and setup uncertainty; they also cover the clinical target volume, which serves to ensure the sterilization of any subclinical microscopic extensions surrounding the GTV. To avoid the risk of disease recurrence, PTV margin reduction can only be safely accomplished at a point when sufficient radiation has been delivered to eliminate invisible microscopic spread. Two possible treatment strategies can be followed after adapting a radiotherapy plan with reduced PTV margins. The first is to reduce healthy tissue irradiation as much as possible while leaving the prescription dose to the target volume unchanged. The second choice is to escalate the dose to the tumor as high as possible while not surpassing the levels of healthy tissue irradiation achieved in the initial plan. The aim of this study is to demonstrate the potential benefits of adaptive radiation therapy by describing a case of mesothelioma with mediastinal metastasis treated using helical tomotherapy. This study evaluates dosimetric planning and tumor volume changes observed on MVCT studies. By using the Planned Adaptive software (TomoTherapy Inc., Madison, WI) to delineate targets and sensitive structures, PTV margins were appropriately reduced, and adapted plans for both treatment strategies were generated. The study quantifies volumetric and dosimetric changes, examines the effects of the adapted plans, and compares them to the original plan. Plans created using alternative treatment modalities (3D conformal radiation therapy [3DCRT] and step-and-shoot IMRT) are also evaluated and compared to original HT plans.

\section{METHODS AND MATERIALS}

A 73-year-old female patient with history of prior exposure to asbestos presented with symptomatic biphasic mesothelioma of the right lobe pleura and a mediastinal mass. The patient had a previous renal lesion 2 years prior, which was stable. Pulmonary function tests were normal. A biopsy sample showed it as a malignant mesothelioma. By the T3N2M0 staging and surgical assessment based on a computed tomography (CT) scan, the disease was confirmed as inoperable. The patient did not receive any chemotherapy and it was decided to give radiotherapy as treatment. A kVCT study (Fig. 1a) was
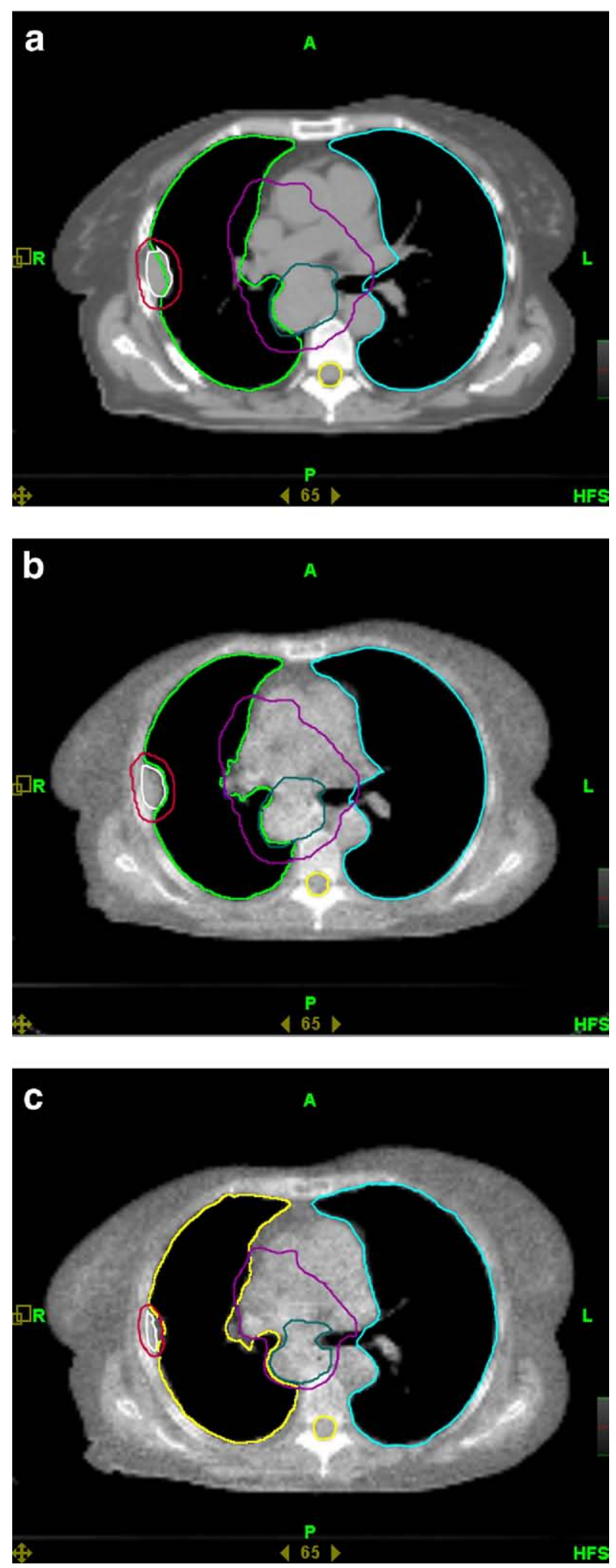

Fig. 1. (a) kVCT image taken 9 days before treatment began. MVCT image acquired prior to (b) 1st fraction and (c) 22nd fraction. $\mathrm{GTV}_{2}$ (green), $\mathrm{GTV}_{1}$ (white), $\mathrm{PTV}_{2}$ (purple), $\mathrm{PTV}_{1}$ (red), ipsilateral (light green), contralateral lungs (light blue), and spinal cord (yellow) are outlined. Long-term volume regression is most notable in $\mathrm{GTV}_{1}$. 
taken 9 days before the start of treatment on a helical CT scanner (Philips Brilliance Big Bore, 3-mm-slice thickness, $120 \mathrm{kVp}$ ). The radiation oncologist (ARD) outlined 2 targets (mediastinal nodal $\mathrm{GTV}_{2}$, pleural lesion $\mathrm{GTV}_{1}$ ) and the following sensitive structures using the Pinnacle treatment-planning system (Pinnacle ${ }^{3}$ version $8.0 \mathrm{~d}$; Philips, Fitchburg, WI): lungs, spinal cord, and heart. All critical structures were assumed to be solid organs for dosimetric purposes. Ipsilateral lung was defined as lung minus both GTVs. $\mathrm{GTV}_{2}$ was delineated with the intention of including affected mediastinal lymph nodes. Planning target volumes were created by $5 \mathrm{~mm}\left(\mathrm{PTV}_{1}\right)$ and $10 \mathrm{~mm}\left(\mathrm{PTV}_{2}\right)$ 3D isotropic margins around $\mathrm{GTV}_{1}$ and $\mathrm{GTV}_{2}$, respectively. Clinical target volumes (CTVs) were defined equal to the PTVs. Initial recorded volumes were $228.4 \mathrm{~cm}^{3}$ for $\mathrm{GTV}_{2}$ and $15.2 \mathrm{~cm}^{3}$ for $\mathrm{GTV}_{1}$. Doses of $50 \mathrm{~Gy}$ to $\mathrm{GTV}_{2}$ and $60 \mathrm{~Gy}$ to $\mathrm{GTV}_{1}$ were prescribed in 30 fractions. The patient received 22 fractions, after which, treatment was stopped due to a non-treatmentrelated arm fracture. A repeat $\mathrm{kVCT}$ scan 3 months after the last radiotherapy fraction and clinical examination showed no radiation complications of either the lungs or esophagus, but there was a residual mediastinal nodal disease.

\section{Helical tomotherapy planning}

CT and structure datasets were transferred to the Tomotherapy Hi-ART planning station (version 2.2.2, TomoTherapy Inc., Madison, WI) using the DICOM RT protocol. Control of dose delivery is achieved through 3 parameters: fan-beam thickness, pitch, and modulation factor, described in detail elsewhere. ${ }^{11}$ For all tomotherapy plans in this study, the fan-beam thickness was set to $5.02 \mathrm{~cm}$, with a pitch of 0.287 , and a maximum modulation factor of 3 . Dose calculation grid was approximately $4 \times 4 \times 3 \mathrm{~mm}^{3}$ Optimization was guided using precedence, importance, and penalty factor parameters, as illustrated in Table 1 . Coverage of $95 \%$ of the PTVs was set as the optimization target in each plan. Desired dosimetric constraints were $\mathrm{D}_{\max }$ for the spinal cord (46 Gy), $D_{30}$ to the heart (45 Gy), as well as combined mean lung dose (20 Gy). About 200 iterations were used in the optimization procedure for each plan after beamlet calculation, with the total planning time (range: $2-4$ hours) depending on the PTV volume. The

Table 1. Optimization parameters for plan 1

\begin{tabular}{lcccccr}
\hline & & $\begin{array}{c}\text { Max } \\
\text { Dose } \\
\text { Structure }\end{array}$ & $\begin{array}{c}\text { Max } \\
\text { Impose } \\
\text { Implance }\end{array}$ & $\begin{array}{c}\text { DVH } \\
\text { Polume } \\
(\%)\end{array}$ & $\begin{array}{r}\text { DVH } \\
\text { Dose } \\
(\text { Gy })\end{array}$ & $\begin{array}{c}\text { DVH } \\
\text { Pt. } \\
\text { Penalty }\end{array}$ \\
\hline Spinal cord & 1 & 44 & 1 & 1 & 43 & 1 \\
Right lung & 1 & 60 & 1 & 17 & 40 & 1 \\
Left lung & 10 & 50 & 1 & 15 & 5 & 33 \\
Heart & 1 & 50 & 1 & 30 & 30 & 1 \\
PTV $_{1}$ & 10 & 60 & 1 & 95 & 50 & 33 \\
PTV $_{2}$ & 10 & 50 & 1 & 95 & 60 & 33 \\
\hline
\end{tabular}

HT plan based on initial anatomy from the kVCT study (plan 1: expected beam-on time: 220.6 seconds per fraction) was approved for treatment.

\section{Delineation of structures}

Daily MVCT studies (Fig. 1b and 1c) were acquired preceding each treatment and were transferred to the planning station. GTVs and OAR contours were adapted for all treatment days using Planned Adaptive to assess volumetric and dosimetric variations. Absolute volumes of the GTVs, as well as $D_{1}$ and $D_{99}$ for the PTVs, were recorded. $\mathrm{D}_{\mathrm{x}}$ denotes dose to $\mathrm{x} \%$ of the structure's volume. Additionally, absolute volume, $\mathrm{V}_{5}, \mathrm{~V}_{10}, \mathrm{~V}_{20}$, and $\mathrm{V}_{30}$ were tabulated for both the contralateral and ipsilateral lungs. $\mathrm{V}_{\mathrm{y}}$ means relative volume of the structure irradiated to y Gy or more. To assess the intraobserver contouring uncertainty, contour adaptation was twice repeated to include both the largest and smallest imaginable GTV volumes on the 1st, 11th, and 23rd fractions. Interpolation of volumetric discrepancies between these days was assumed to be linear.

\section{Reducing healthy tissue irradiation}

After 22 fractions, the MVCT studies showed observable volume reduction in both GTVs. At this point, it was assumed that the subclinical microscopic disease of the target volumes had been sterilized allowing for safe reduction of both PTV margins to $4 \mathrm{~mm}$. Our hypothesis is that microscopic tumor spread is relatively more radiosensitive and well oxygenated, so that likelihood of these tumor cells survival and/or regrowth is very low after irradiation during 22 fractions (2 Gy/fraction). These reduced margins would be sufficient to account for post image-guided alignment setup errors and intrafraction movement. An adapted plan with updated structure outlines to reflect the anatomy changes was generated using the MVCT study taken on the 22nd fraction (plan 2: beam-on time 229.0 seconds). All optimization parameters were left unchanged from plan 1 for comparative purposes. This plan represented the first of the 2 treatment strategies; it kept the original prescribed dose to the targets and reduced the critical organ dose.

\section{Escalating dose}

A third plan reflecting the second strategy, that is, to increase the target dose while maintaining the same level of healthy tissue sparing achieved in plan 1, was also created (plan 3: beam-on time 256.6 seconds). The same structure and image sets as in plan 2 were used. Increased prescription doses were determined as follows: in plan 1, the percentage of ipsilateral lung receiving $20 \mathrm{~Gy}$ or more $\mathrm{V}_{20}$ was approximately $80.5 \%$ and, in plan 2 , the same percentage was receiving about $17 \mathrm{~Gy}$. The ratio of these values $(\sim 1.175)$ was applied as a scaling factor to the original PTV doses. The intent was to generate a plan in which the ipsilateral lung $\mathrm{V}_{20}$ was equivalent to that of plan 1 . The choice of $\mathrm{V}_{20}$ as a reference point was based 
upon its clinical significance as a risk indicator for acute toxicity development. ${ }^{20}$ With the exception of the spinal cord, dose constraint parameters used by the planning software were also scaled by the same value. The spinal cord exceeded its clinically accepted maximum dose when scaled; therefore, it was left unchanged from plan 1 .

\section{Dose quality factor}

As lung cancer is one of the more difficult diseases to treat with radiotherapy, it is advantageous to compare different available radiation delivery modalities. Plan 1 was reproduced using both step-and-shoot IMRT and 3DCRT. These alternative solutions were subsequently compared to HT using a dose quality factor (DQF) scoring option. The DQF is a single value assigned to each treatment plan based on a balanced scoring scheme for target coverage and normal tissue sparing. ${ }^{21}$ It is defined as:

$$
\mathrm{DQF}=\mathrm{DQF}(\text { target }) \times \mathrm{DQF}(\mathrm{OAR})
$$

DQF for target and OAR calculations are based on the acceptable dose $\left(D_{a c c}\right)$ as well as the actually achieved dose $(D)$ for selected sub-volumes:

$$
\begin{aligned}
& \mathrm{DQF}(\text { target })=\sum w_{i}\left(\left(\frac{D}{D_{a c c}}\right)^{n}\right)_{i} \\
& \mathrm{DQF}(\mathrm{OAR})=\sum w_{j}\left(\left(\frac{D_{a c c}}{D}\right)^{n}\right)_{j}
\end{aligned}
$$

where $w$ is the weighting for a specific structure and $i$ and $j$ are indices that sum over all targets and OARs, respectively. The sum over all weighting factors is one. For the $\mathrm{DQF}$ (target), $D / D_{a c c}$ is set to unity if $D>D_{a c c}$. In the case of DQF(OAR), the same is done if $D<D_{a c c}$. The exponent $n$, in Eqs. (2) and (3), allows for different dose levels to have differently assigned importance factors. Table 2 lists the acceptable doses, subvolumes, weighting factors, and values of $n$ used in this comparative planning study for each structure. These values have been chosen based on the relative importance of meeting the required dose for different structures, as seen through clinical experience. $^{21}$

\section{RESULTS AND DISCUSSION}

In this study, initial (and clinically applied) plan 1 and two "adapted" treatment plans, one aimed to improve OAR sparing (plan 2) and another to escalate target dose (plan 3), were generated. Dose-volume histograms (DVHs) were analyzed to compare different possible treatment options and are shown in Fig. 2. Sensitive structure tolerance dose limits were met for the heart in all plans, for the lungs in plans 2 and 3, and for the spinal cord in plan 2 (Table 3 ). In plan 1, combined mean lung dose (MLD) was $20.22 \mathrm{~Gy}$. This high dose to the lungs is due to this patient's specific anatomy, with 2 targets far apart and HT's helical mode of radiation delivery. $\mathrm{PTV}_{2}$ is very close to the spinal cord and only the reduction of PTV margins in plan 2 could effectively decrease the cord's $\mathrm{D}_{\max }$ below $46 \mathrm{~Gy}$.

\section{Plan 2 (improved OAR sparing)}

$\mathrm{PTV}_{2}$ was reduced from 595 to $320 \mathrm{~cm}^{3}$ and $\mathrm{PTV}_{1}$ from 45.8 to $25.4 \mathrm{~cm}^{3}$ after 22 fractions. The percentage of ipsilateral lung $\mathrm{V}_{20}$ was reduced by $7.9 \%$ in plan 2. Similarly, planned mean dose delivered to the ipsilateral lung was decreased from 34.1 to $28.5 \mathrm{~Gy}$. The planned combined lung MLD decreased by 3.93 Gy and $\mathrm{V}_{20}$ by $5.4 \%$. Because this plan would be applied to the final 8 fractions of treatment only, combined lung MLD is actually lowered by 1.05 Gy and $\mathrm{V}_{20}$ by $1.4 \%$. Dose distributions for plans 1 and 2 are shown in Figs. 3 and 4, respectively. Both plans demonstrate high conformality of the 50 and 60 Gy isodose lines to their respective target contours. Figure 5 shows a comparison of DVHs for plans 1 and 2. For all OARs, the sparing of healthy tissue is improved in plan 2 (most notably for the ipsilateral lung), while providing a comparable target volume coverage.

\section{Plan 3 (escalated prescription dose)}

Prescribed doses to $\mathrm{PTV}_{2}$ and $\mathrm{PTV}_{1}$ were scaled by a

\begin{tabular}{|c|c|c|c|c|c|}
\hline & Parameter & $\begin{array}{c}\text { Acceptable Dose Level } \\
\text { (Gy) }\end{array}$ & Subvolume (\%) & Weighting & $n$ \\
\hline Target 1 & $\mathrm{PTV}_{2}$ minimum dose & $43.7^{\mathrm{a}}$ & 99 & 0.4 & 1 \\
\hline Target 2 & $\mathrm{PTV}_{1}$ minimum dose & $57.0^{\mathrm{a}}$ & 99 & 0.4 & 2 \\
\hline Target 3 & $\mathrm{PTV}_{1}$ prescription & 60.0 & 95 & 0.15 & 1 \\
\hline Target 4 & $\mathrm{PTV}_{1}$ maximum dose & $64.2^{\mathrm{b}}$ & 1 & 0.05 & -1 \\
\hline OAR 1 & Mean lung dose & 20.0 & $\mathrm{n} / \mathrm{a}$ & 0.4 & 1 \\
\hline OAR 2 & Spinal cord $\mathrm{D}_{\max }$ & 46.0 & 0 & 0.4 & 2 \\
\hline OAR 3 & Heart $D_{30}$ & 45.0 & 30 & 0.1 & 1 \\
\hline OAR 4 & Esophagus $D_{1}$ & 60.0 & 1 & 0.1 & 1 \\
\hline
\end{tabular}
factor of 1.175 , increasing them from 50 and $60 \mathrm{~Gy}$ to 58.75

Table 2. Parameters used for calculation of the dose quality factor

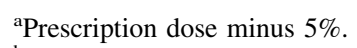

bPrescription dose plus $7 \%$. 


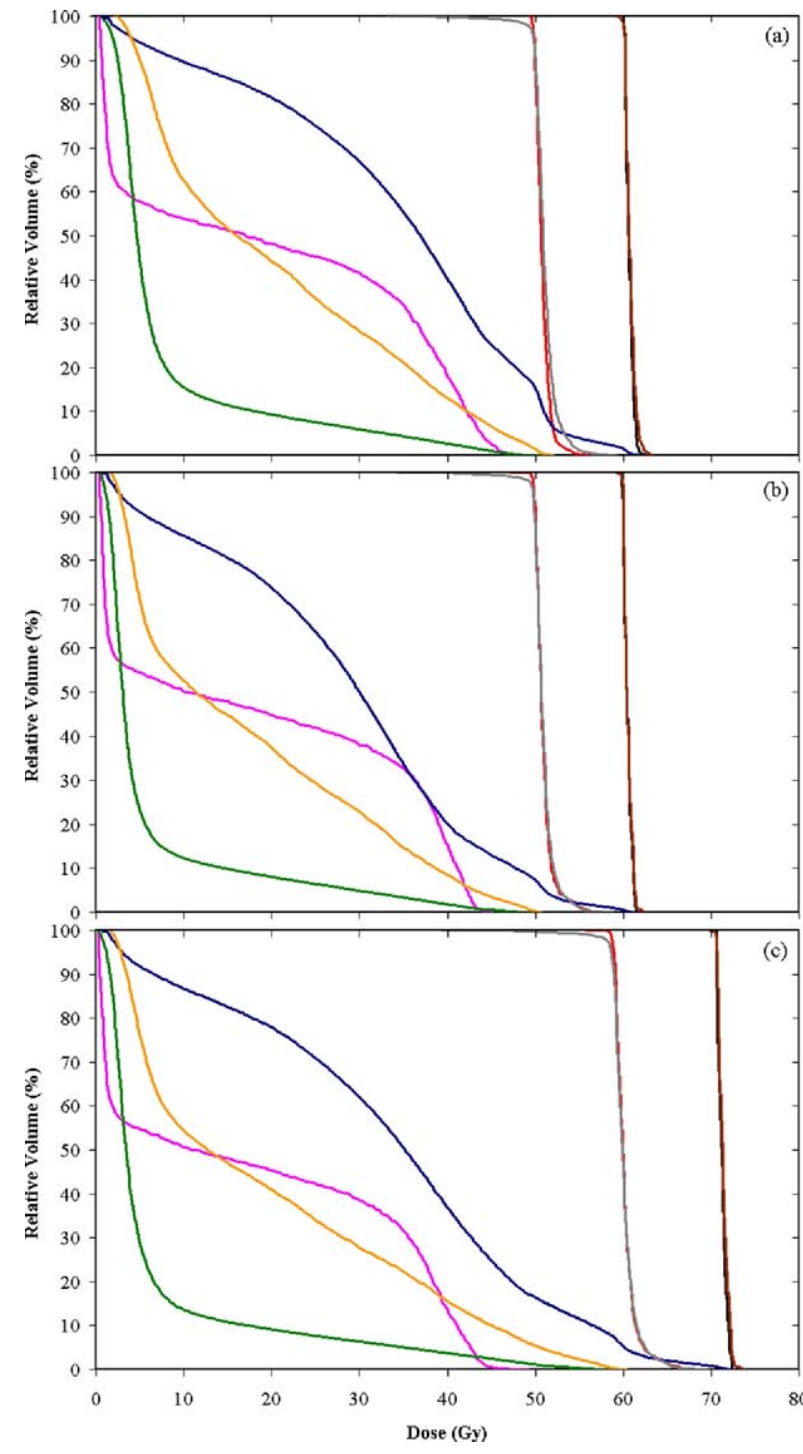

Fig. 2. (a) DVH of plan 1 for HT delivery. Color code: dark blue $=$ ipsilateral lung; light blue $=$ contralateral lung; green $=$ heart; orange $=$ spinal cord; red $=\mathrm{GTV}_{2}$; pink $=\mathrm{PTV}_{2}$; grey $=\mathrm{GTV}_{1}$; black $=\mathrm{PTV}_{1}$. (b) DVH of plan 2. (c) DVH of plan 3.

and $70.5 \mathrm{~Gy}$, respectively. This approach aimed to increase the prescribed dose to achieve the same ipsilateral lung $\mathrm{V}_{20}$ value as in plan 1 . Our calculation of $\mathrm{V}_{20}$ value for plan 3 resulted in $77.8 \%, 3.7 \%$ less than the original plan 1 . If the plan were applied to the
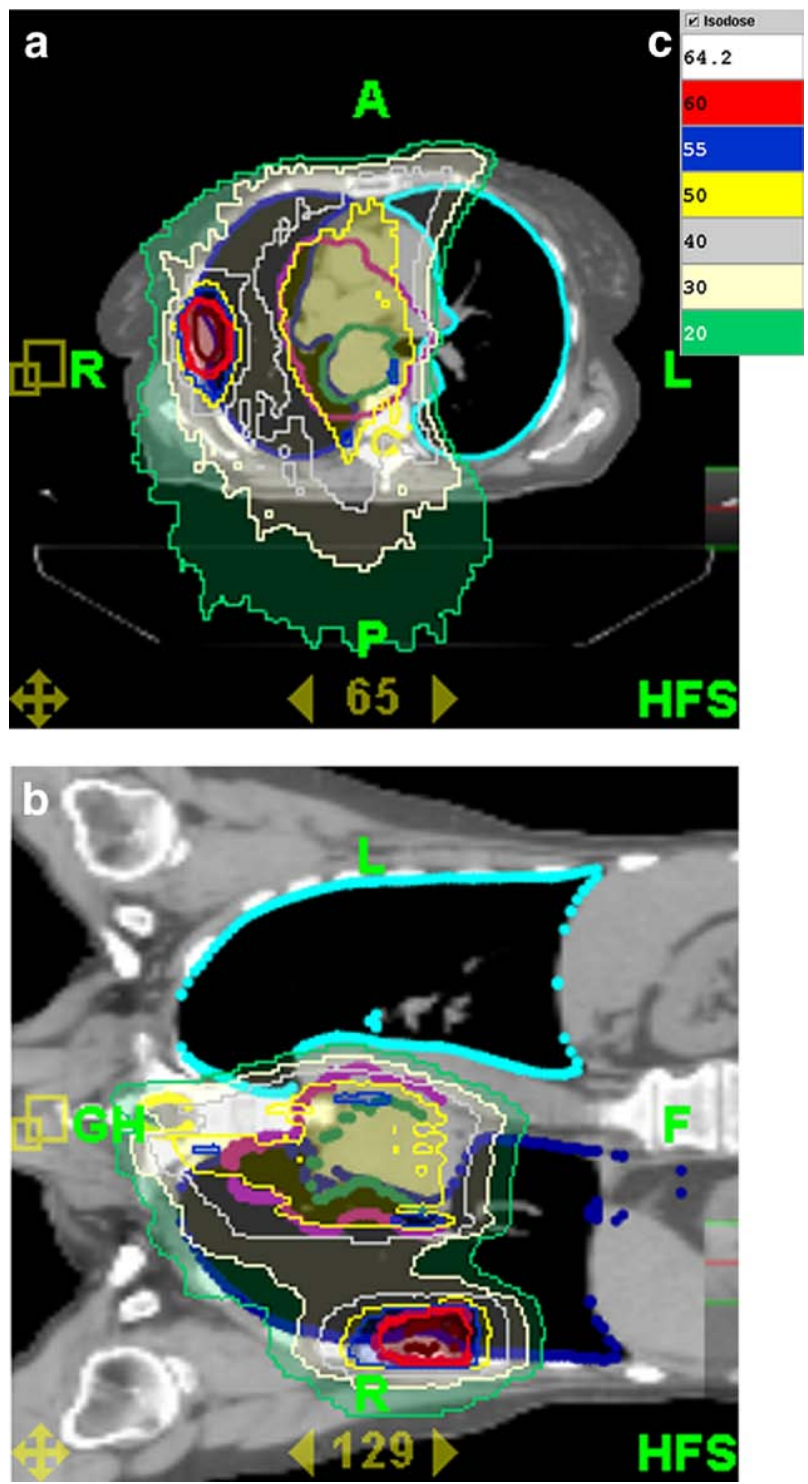

Fig. 3. Dose distribution (a) axial and (b) sagittal produced for plan 1 on the 33rd day of treatment. (c) Color code for isodose lines.

remaining 8 fractions, then the dose to $\mathrm{PTV}_{2}$ and $\mathrm{PTV}_{1}$ would be increased to 52.3 and $62.8 \mathrm{~Gy}$, respectively. Martel et ll. $^{22}$ estimated the effects of total radiation dose on probability of tumor control and found that when 65 Gy was delivered, $53 \%$ and $26 \%$ of patients experienced local progression-free survival after 12

Table 3. Summary of tomotherapy plans

\begin{tabular}{|c|c|c|c|c|c|c|c|c|}
\hline \multirow[b]{2}{*}{ Plan } & \multicolumn{2}{|c|}{$\mathrm{PTV}_{2}$} & \multicolumn{2}{|c|}{$\mathrm{PTV}_{1}$} & \multicolumn{2}{|c|}{ Lungs } & \multirow[b]{2}{*}{ Cord $D_{\max }(G y)$} & \multirow[b]{2}{*}{ Heart $\mathrm{D}_{30}(\mathrm{~Gy})$} \\
\hline & $\mathrm{D}_{99}(\mathrm{~Gy})$ & $\mathrm{D}_{1}(\mathrm{~Gy})$ & $\mathrm{D}_{99}(\mathrm{~Gy})$ & $\mathrm{D}_{1}(\mathrm{~Gy})$ & $\mathrm{V}_{20}(\%)$ & MLD (Gy) & & \\
\hline 1 & 47.37 & 55.36 & 59.97 & 62.56 & 43.51 & 20.22 & 49.11 & 28.87 \\
\hline 2 & 47.45 & 55.02 & 59.86 & 61.64 & 38.12 & 16.29 & 45.18 & 24.42 \\
\hline 3 & 56.04 & 65.24 & 70.49 & 72.69 & 40.55 & 19.07 & 49.23 & 28.06 \\
\hline
\end{tabular}

Abbreviations: MLD, mean lung dose; $\mathrm{D}_{\mathrm{x}}$, dose to $\mathrm{x} \%$ of the organ volume; $\mathrm{V}_{20}$, relative volume exposed to $\geq 20$-Gy dose; $\mathrm{D}_{\max }$, maximum dose to organ. 

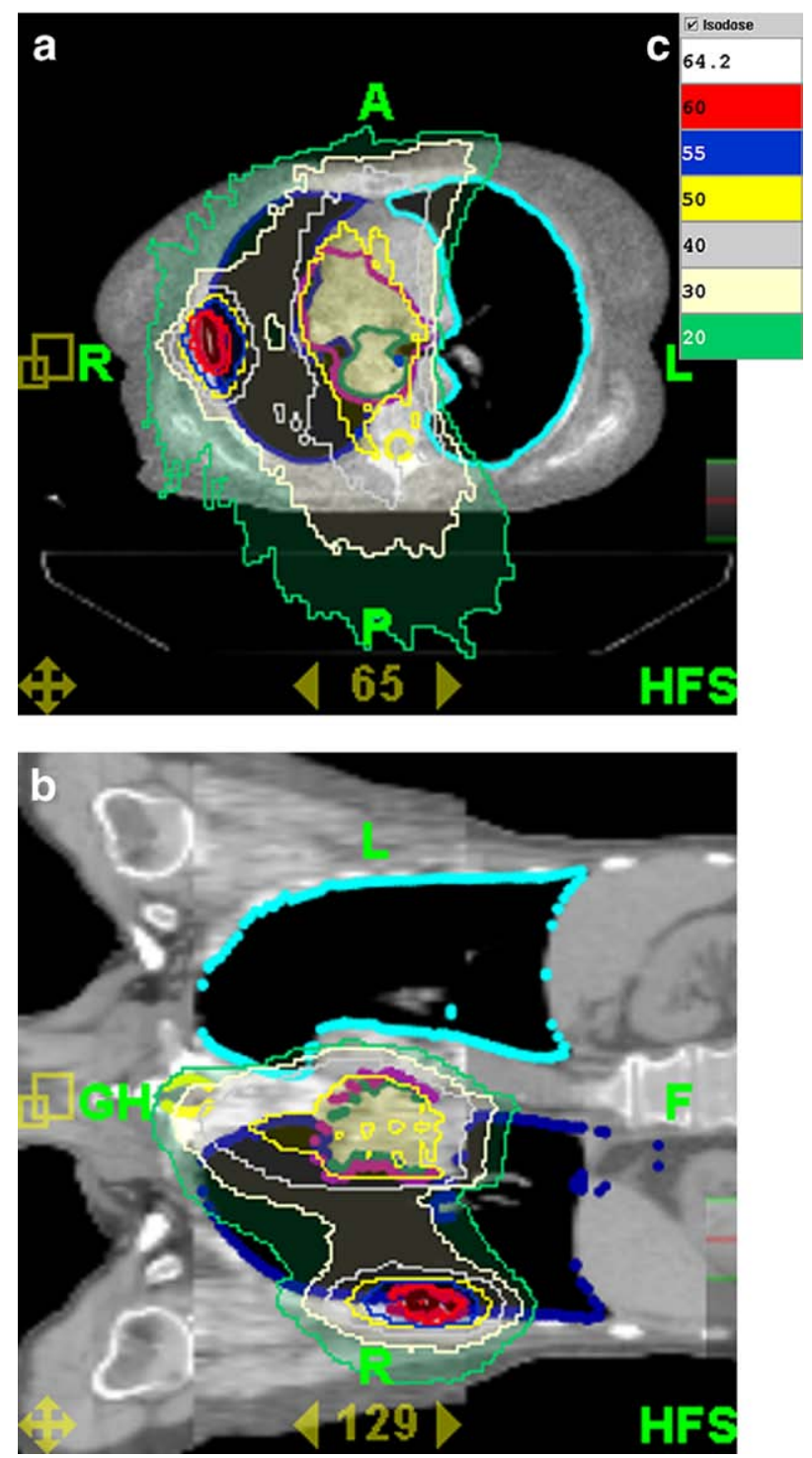

Fig. 4. (a) Axial and (b) sagittal dose distributions that would have been produced for plan 2 on the 33rd day of treatment. (c) Color code for isodose lines.

and 30 months, respectively. Examining the dosimetric data set of plan 3 reveals a general improvement in healthy lung sparing, despite the increase doses to targets due to the decreased target margins. The MLD and $\mathrm{V}_{20}$ for both lungs are $5.7 \%$ and $6.8 \%$ lower than in the original plan. Prescription isodose lines again conform nicely to the target volumes, as seen in Fig. 6. Relative homogeneity of dose distributions for both target volumes is achieved. In this case, the proximity of the heart was the limiting factor for dose escalation. Due to the heart's slight overlap with the GTV, its $\mathrm{D}_{\max }$ was raised to $60.3 \mathrm{~Gy}$ in plan 3. Had the positioning of the heart not been an issue, further escalation of the prescribed doses could have been potentially feasible. The escalation of dose presents an issue in fractionation that needs to be addressed. One option is to increase number of fractions needed to deliver this dose proportionally, keeping the dose per fraction unchanged from the original plan. The other possibility would be to keep the existing number of remaining fractions and increase the dose per fraction. The biological effect of these options is not the same and, in fact, reducing the overall treatment time has been shown to have favorable effects on survival. Saunders et $a l .^{23}$ performed a study comparing accelerated vs. conventional radiotherapy in patients with non-small cell lung cancer and found that the accelerated patients showed improved survival rates $(63 \%$ vs. $55 \%$ after 1 year, $29 \%$ vs. $20 \%$ after 2 years, and $20 \%$ vs. $13 \%$ after 3 years).

\section{Tumor regression}

A total of 23 MVCT imaging sessions were performed over the span of 36 days. During that time, there was a $16.2 \%$ and $32.5 \%$ measured volume reduction in $\mathrm{GTV}_{2}$ and $\mathrm{GTV}_{1}$, respectively. Relative changes in $\mathrm{GTV}_{2}$ and $\mathrm{GTV}_{1}$ over the course of treatment are presented in Fig. 7. Mean volume change per day for the $\mathrm{GTV}_{2}$ was $-0.7 \% \pm 3.5 \%$, with a range of $-7.5 \%$ to $7.8 \%$ change per day. For $\mathrm{GTV}_{1}$, the mean volume change was $-2.0 \% \pm 10.9 \%$, with a range of $-25 \%$ to $27 \%$ change per day. In both cases, tumor regression was generally a very slow process, with no periods of accelerated decreasing. If these results are similar to the results of Woodford et al., GTV Gould $_{1}$ fall into group B (a more gradual linearly decreasing tumor volume), whereas $\mathrm{GTV}_{2}$ would most likely fall into group $\mathrm{C}$ (experienced variable volume changes with no clear trend toward a decrease in volume). ${ }^{17}$ This information on tumor regression does not imply that regression will stop once the therapy is through. In fact, it is shown that NSCLC tumors reach their

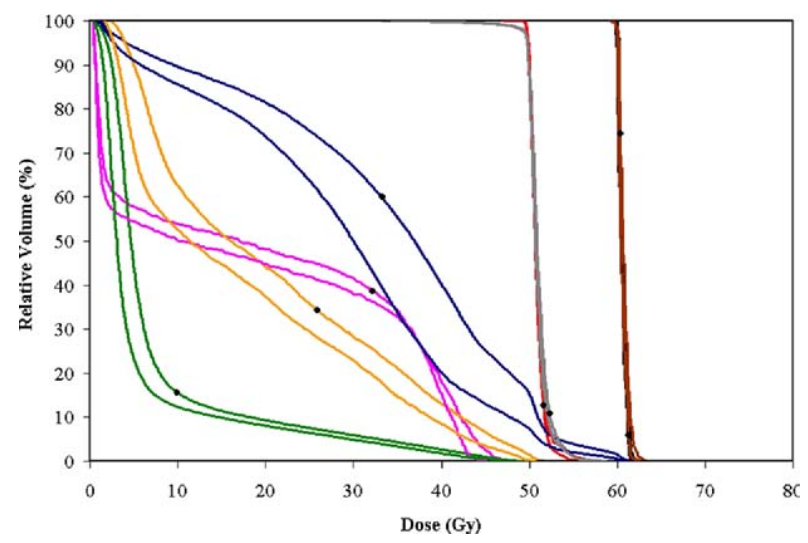

Fig. 5. DVH of plan 1 and plan 2. Lines from plan 1 have black circles on them. It is clear that plan 2 spares more healthy tissue while providing virtually the same target coverage. Color code: dark blue $=$ ipsilateral lung; light blue $=$ contralateral lung; green $=$ heart; orange $=$ spinal cord; grey $=\mathrm{GTV}_{2}$; pink = $\mathrm{PTV}_{2} ;$ black $=\mathrm{GTV}_{1} ;$ red $=\mathrm{PTV}_{1}$. 

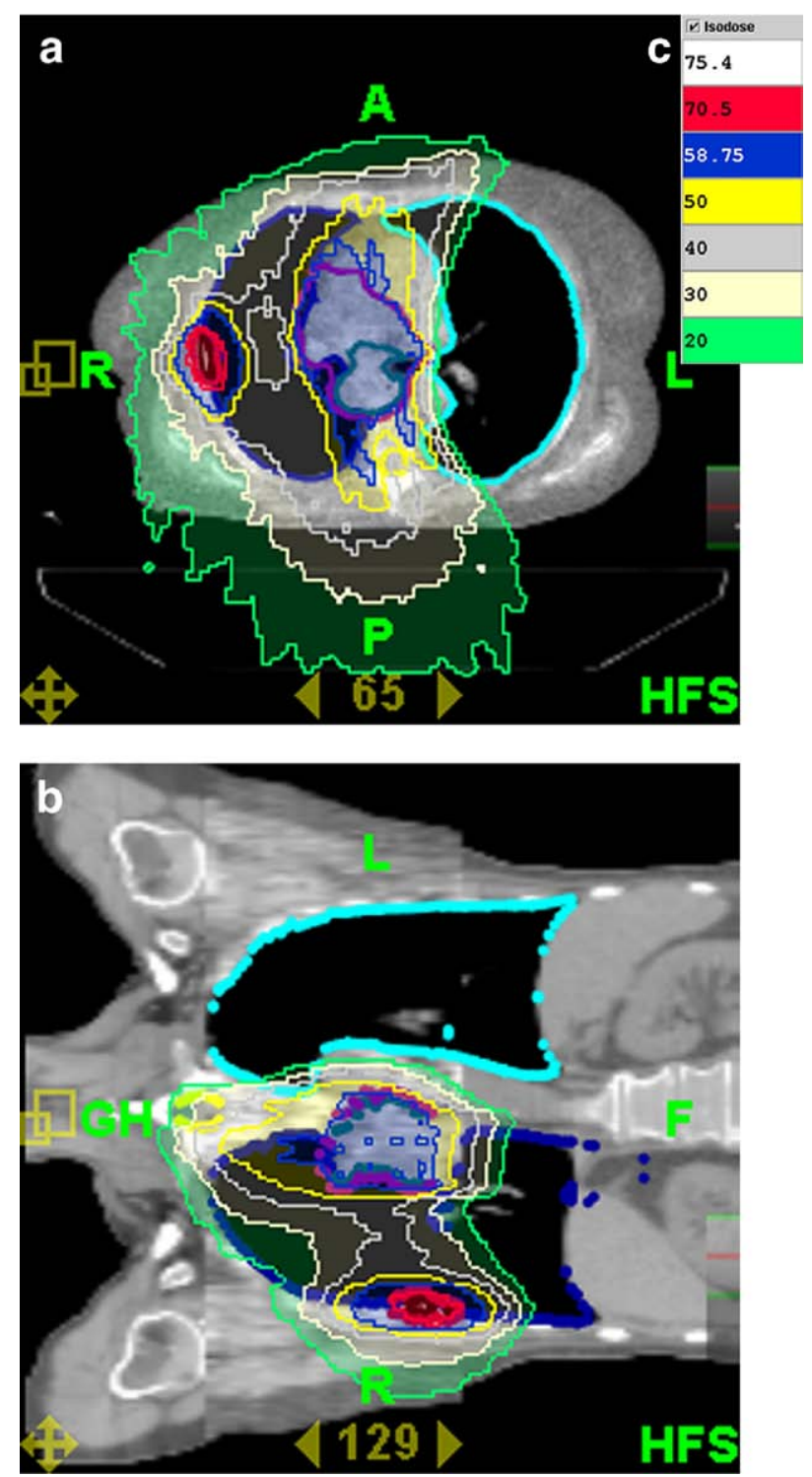

Fig. 6. (a) Axial and (b) sagittal dose distributions that would have been produced for plan 3 on the 33rd day of treatment. (c) Color code for isodose lines.

minimum volume, on average, 11 months after radiation completion. ${ }^{24}$ According to classification of tumor regression proposed by Siker et al., the responses of both GTVs would be stable $(0-34 \%$ regression $){ }^{4}$ Applying these criteria to the observed GTV changes on HT by several groups, ${ }^{4,16,17}$ the frequency of stable responses vary. In 2 data sets, Siker et al. reported $68 \%$ and $53 \%$ of patients having stable tumor responses, whereas Woodford et al. similarly observed $41 \% .^{4,17}$ This contrasts the work of Kupelian et al., who reported no stable responses in any patients. ${ }^{16}$

\section{Contouring uncertainty}

With each slice reconstructed from a 5-second data acquisition, MVCT is a "slow" CT scan, with some averaging of moving objects. Tumor volume and position uncertainty is directly related to the patient's breathing cycle, which, in turn, is largely dependant upon the level of comfort. ${ }^{25}$ A large amount of uncertainty in delineating $\mathrm{GTV}_{2}$ was associated with it being primarily contained within the mediastinum. ${ }^{26}$ The MVCT image sets used to contour $\mathrm{GTV}_{2}$ did not have sufficient contrast and resolution to reliably adapt the contours contained within the mediastinum. $\mathrm{GTV}_{1}$, on the other hand, is considered to be a peripherallung tumor and it was easily detected and delineated on the MVCT image sets. ${ }^{26}$

\section{Patient positioning}

In addition to the detection of any significant interfraction anatomy changes, such as tumor regression or position shift, MVCT studies enable the correction of patient positioning just prior to treatment. Throughout this patient treatment, the average positioning corrections (in $\mathrm{mm}$ ) in the $\mathrm{x}$ (lateral), $\mathrm{y}$ (superior-inferior), and $\mathrm{z}$ (anterior-posterior) directions were $2.7 \pm 2.7,0.8 \pm 2.9$, and $8.0 \pm 3.2$, respectively. There are several factors that may influence the position correction. This patient experienced significant weight loss over the course of treatment. External marking tattoos used for initial setup were most likely displaced with respect to the internal organs. Also, pain and difficulty in breathing often pre-

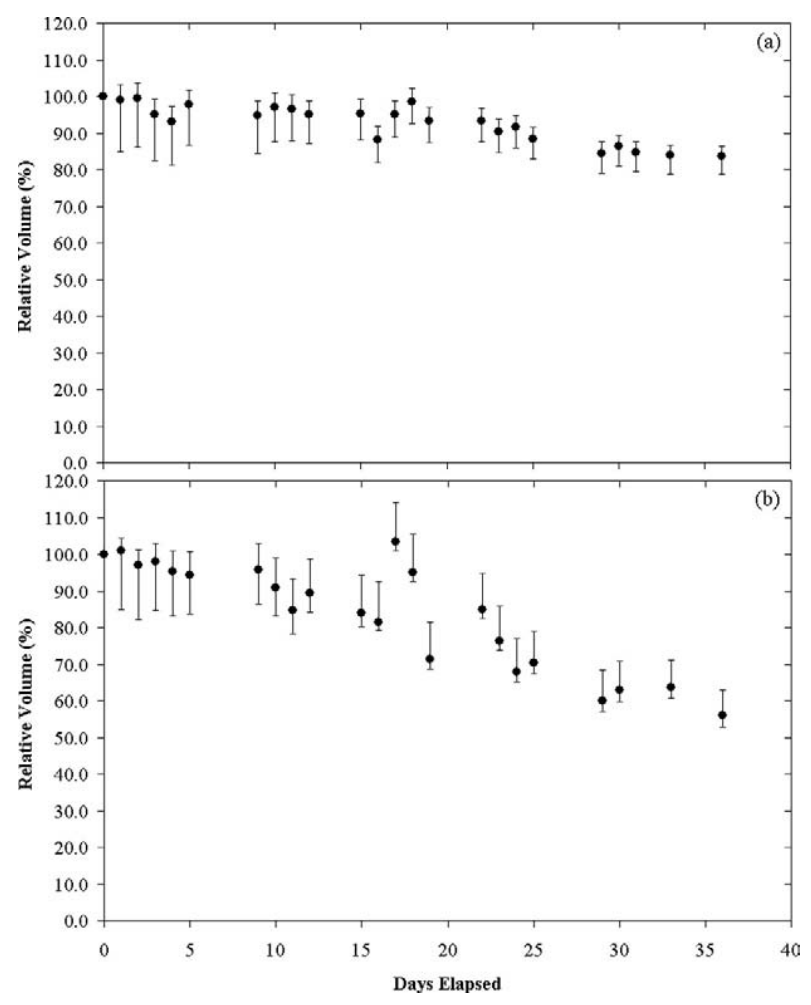

Fig. 7. (a) Relative $\mathrm{GTV}_{2}$ and (b) $\mathrm{GTV}_{1}$ volumes recorded over the course of treatment. 
vent patients from keeping their hands up over their head in the same position, as required during treatment. In the $\mathrm{z}$ direction, the average positional shift is around $8 \mathrm{~mm}$. This can be explained by the mechanical differences in the planning $\mathrm{kVCT}$ and the MVCT couches; the latter is more flexible. The corrective shifts in the $y$ direction alternate from negative to positive over the course of treatment with respect to zero. The average shift in the lateral direction is due to the GTV changes most prominent in this direction.

\section{Step-and-shoot IMRT plan for this patient}

A step-and-shoot IMRT plan was created with the Pinnacle planning system for the same planning kVCT study and organ contours and was compared to the original HT plan. Three 6-MV beams and two 18-MV beams were chosen to achieve a reasonable coverage of the target volumes while avoiding OAR irradiation as much as possible. The dose distribution and DVH curves for the step-and-shoot IMRT plan are shown in
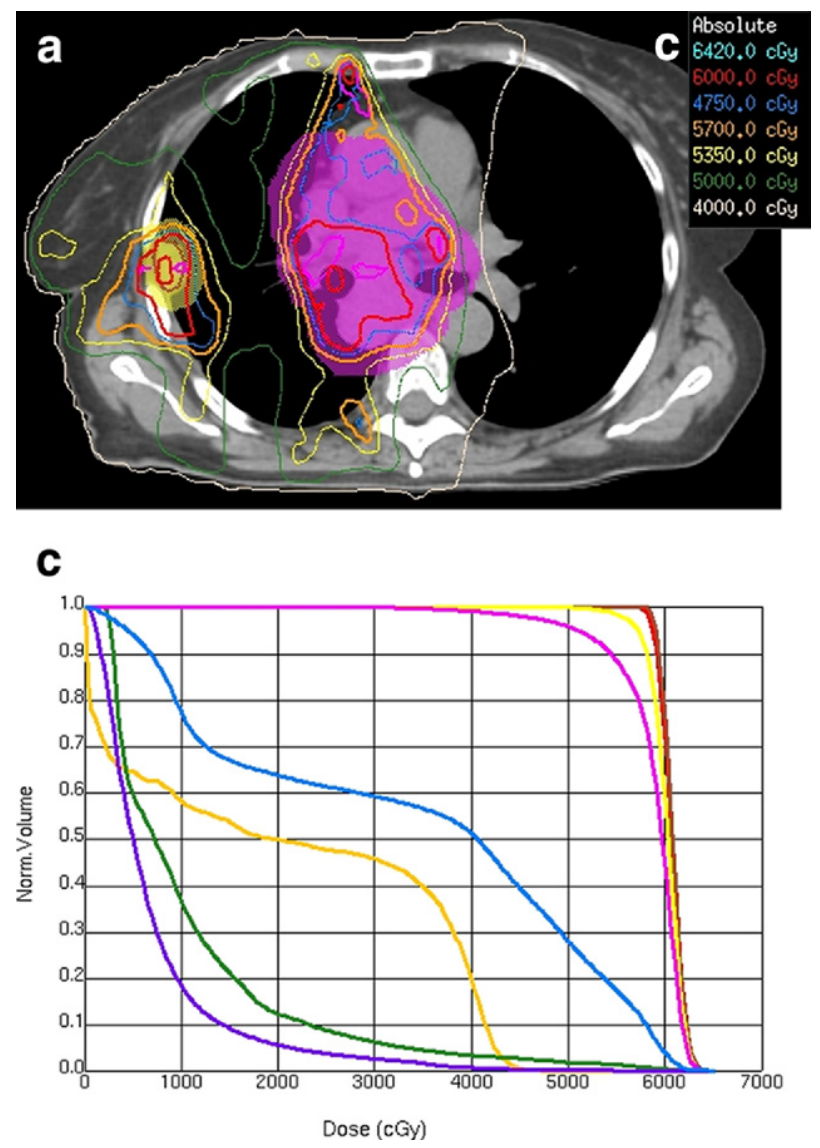

Fig. 8. (a) Axial dose distribution that would have been produced by radiation fluence of the step-and-shoot IMRT version of plan 1. (b) Color code for isodose lines. (c) DVH of stepand-shoot IMRT plan. Color code: light blue = ipsilateral lung; dark blue $=$ contralateral lung; red $=$ heart; orange $=$ spinal cord; red $=\mathrm{GTV}_{2} ;$ purple $=\mathrm{PTV}_{2}$; brown $=\mathrm{GTV}_{1}$; yellow $=$ $\mathrm{PTV}_{1}$.

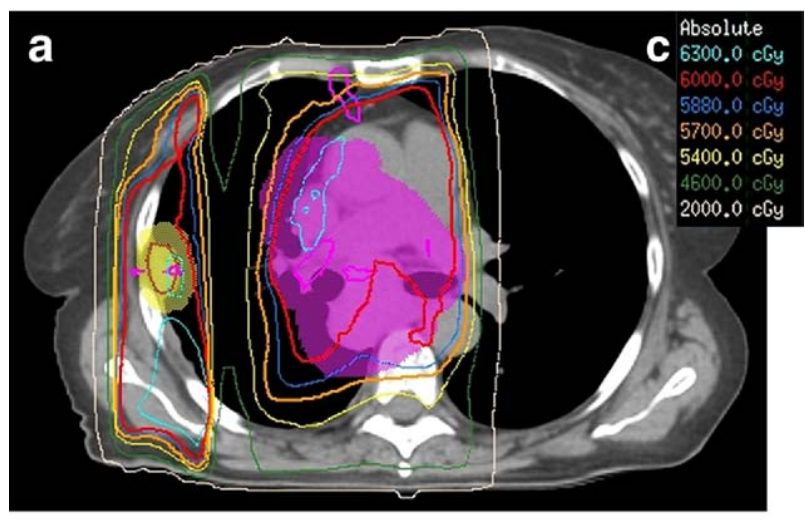

C

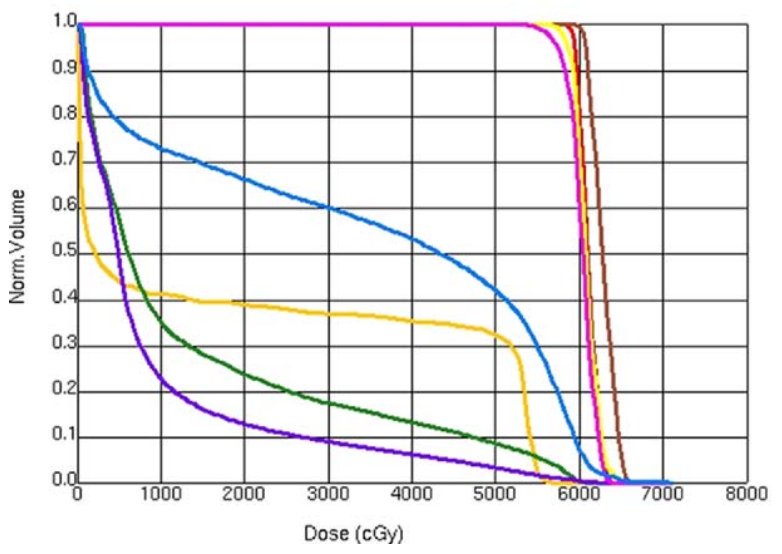

Fig. 9. (a) Axial dose distribution that would have been produced by radiation fluence of the 3DCRT version of plan 1 . (b) Color code for isodose lines. (c) DVH of 3DCRT plan. Colour code: light blue $=$ ipsilateral lung; dark blue $=$ contralateral lung; red = heart; orange = spinal cord; red $=\mathrm{GTV}_{2}$; purple $=\mathrm{PTV}_{2}$; brown $=\mathrm{GTV}_{1}$; yellow $=\mathrm{PTV}_{1}$.

Fig. 8 (a) and (c). An examination of the DVHs reveals an overall improvement in healthy tissue sparing over the original HT plan. Significant gains include the heart $\mathrm{D}_{30}$ being reduced to $11.5 \mathrm{~Gy}$ (HT plan: 28.9 Gy), combined lung $\mathrm{V}_{20}$ and MLD lowered to $33.3 \%$ and 19.8 Gy (HT plan: $43.5 \%$ and $20.2 \mathrm{~Gy}$ ), and $\mathrm{D}_{\max }$ to the spinal cord decreased to 47.2 Gy (HT plan: 49.1 Gy). Consequently, this plan has a better DQF(OAR) (see Eq. [3]) score than HT (0.958 vs. 0.938). However, an examination of target coverage shows that the step-and-shoot IMRT plan does not deliver as conformal a dose distribution as does HT. This is exemplified in $\mathrm{D}_{99}$ and $\mathrm{D}_{95}$ values for both of the targets. The HT plan delivered 59.3 and 59.9 Gy to $99 \%$ and $95 \%$ of $\mathrm{PTV}_{1}$, respectively, whereas corresponding step-andshoot IMRT plan values were lower-53.8 and 57.1 Gy. Furthermore, $99 \%$ of $\mathrm{PTV}_{2}$ received 39.7 Gy using the step-and-shoot IMRT plan, much lower than the 45.5 Gy provided by HT. Due to the scoring scheme's heavy weighting of the target $\mathrm{D}_{99}$ statistic, step-andshoot IMRT's DQF(target) score is notably lower than that of HT (0.883 vs. 0.983). Overall DQF scores place 
Table 4. Dosimetric comparison of different treatment modalities

\begin{tabular}{|c|c|c|c|c|c|}
\hline & Parameter & $\begin{array}{c}\text { Acceptable } \\
\text { Dose Level } \\
\text { (Gy) }\end{array}$ & $\begin{array}{l}\text { HT } \\
\text { (Gy) }\end{array}$ & $\begin{array}{l}\text { IMRT } \\
\text { (Gy) }\end{array}$ & $\begin{array}{c}\text { 3DCRT } \\
\text { (Gy) }\end{array}$ \\
\hline Target 1 & $\begin{array}{l}\mathrm{PTV}_{2} \text { minimum } \\
\text { dose }\end{array}$ & $47.5^{\mathrm{a}}$ & 45.5 & 39.7 & 55.09 \\
\hline Target 2 & $\begin{array}{l}\mathrm{PTV}_{1} \text { minimum } \\
\text { dose }\end{array}$ & $57.0^{\mathrm{a}}$ & 59.3 & 53.8 & 57.40 \\
\hline Target 3 & $\mathrm{PTV}_{1}$ prescription & 60.0 & 59.9 & 57.1 & 58.70 \\
\hline Target 4 & $\begin{array}{l}\mathrm{PTV}_{1} \text { maximum } \\
\text { dose }\end{array}$ & $64.2^{\mathrm{b}}$ & 62.0 & 63.0 & 64.75 \\
\hline OAR 1 & Mean lung dose & 20.0 & 20.2 & 19.8 & 21.40 \\
\hline OAR 2 & Spinal cord $D_{\max }$ & 46.0 & 49.1 & 47.2 & 56.70 \\
\hline \multirow[t]{4}{*}{ OAR 3} & Heart $D_{30}$ & 45.0 & 28.9 & 11.5 & 13.20 \\
\hline & $\mathrm{DQF}$ (target) & & 0.938 & 0.958 & 0.862 \\
\hline & DQF(OAR) & & 0.983 & 0.837 & 0.996 \\
\hline & $\mathrm{DQF}$ (overall) & & 0.922 & 0.801 & 0.857 \\
\hline
\end{tabular}

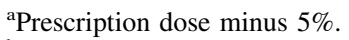

${ }^{\mathrm{b}}$ Prescription dose plus $7 \%$.

HT (0.922) as a more appropriate treatment solution when compared to the step-and-shoot IMRT plan (0.847).

\section{DCRT plan for this patient}

The Pinnacle planning system was also used to create a 3DCRT plan. Planning with this modality was particularly challenging due to the geometry of the 2 separate targets. When two 6-MV and four 18-MV beams were initially orientated to achieve a reasonable coverage of both PTVs, some critical structures received clinically unacceptable levels of irradiation. Improvement for healthy tissue sparing was accomplished through the use of physical wedges as beam attenuators. The achieved dose distribution and DVH curves are shown in Fig. 9 (a) and (c). Target coverage is comparable and, in some cases, slightly improved over the HT plan, especially for PTV 2 . Using 3DCRT, $99 \%$ of $\mathrm{PTV}_{2}$ would receive 55.1 Gy compared to 45.5 Gy in the HT plan. The DQF(target) for the 3DCRT plan (0.996) is therefore slightly better than HT plan 1 (0.983). Healthy tissue sparing offered by both modalities is, for the most part, also quite comparable. Using 3DCRT, combined lung MLD is slightly higher at 21.4 Gy (HT plan: $20.2 \mathrm{~Gy}$ ), combined lung $\mathrm{V}_{20}$ is lowered to $38.1 \%$ (HT plan: $43.5 \%$ ), and $\mathrm{D}_{30}$ for the heart is significantly reduced to $13.2 \mathrm{~Gy}$ (HT plan: 28.9 Gy). However, a maximum spinal cord dose of 56.7 Gy (HT plan: 49.1 Gy) is too high to be clinically acceptable. Large exiting doses from the beam covering $\mathrm{PTV}_{1}$ were received by the cord. Relatively heavy weighting of the spinal cord gave 3DCRT a DQF(OAR) score of 0.862 , and an overall DQF score of 0.859 . In general, due to the complex target geometry of this case, 3DCRT would not be clinically appropriate without decreasing PTV prescription doses by about $10 \mathrm{~Gy}$ to decrease spinal cord irradiation to acceptable levels. Table 4 sum- marizes the dosimetric parameters used to determine DQF scores.

\section{CONCLUSIONS}

Daily MVCT studies provide information for patient setup correction, which can be used to decrease the PTV margins even before the treatment starts. Further plan improvement can be achieved if the target exhibits a volume reduction as a result of radiation delivery, which should be significant enough to warrant a replanning. The choice of specific adaptation strategy (in our case, dose escalation or improved sparing) is related to the specific anatomy of a particular patient. Daily imaging is not exclusive to HT; alternative treatment modalities such as step-and-shoot IMRT equipped with cone beam CT may provide similar adaptive capabilities. As a result, more comparative planning for a larger patient database should be done to establish guidelines for preferential usage of HT or traditional linear accelerators.

Acknowledgments-This study was supported by the Ontario Institute for Cancer Research through the funding provided by the government of Ontario.

\section{REFERENCES}

1. Miles, E.F.; Larrier, N.A.; Kelsey, C.R.; et al. Intensity-modulated radiotherapy for resected mesothelioma: The Duke experience. Int. J. Radiat. Oncol. Biol. Phys. 71:1143-50; 2008.

2. Krayenbuehl, J.; Oertel, S.; Davis, J.B.; et al. Combined photon and electron three-dimensional conformal versus intensity-modulated radiotherapy with integrated boost for adjuvant treatment of malignant pleural mesothelioma after pleuropneumonectomy. Int. J. Radiat. Oncol. Biol. Phys. 69:1593-9; 2007.

3. Perez, C.A.; Pajak, T.F.; Rubin, P. Long-term observations of the patterns of failure in patients with unresectable non-oat cell carcinoma of the lung treated with definitive radiotherapy: Report of the Radiation Therapy Oncology Group. Cancer 59:1874-81; 1987.

4. Siker, M.L.; Tomë, W.A.; Mehta, M.P. Tumor volume changes on series imaging with megavoltage CT for non-small-cell lung cancer during intensity-modulated radiotherapy: How reliable, consistent, and meaningful is the effect? Int. J. Radiat. Oncol. Biol. Phys. 66:135-41; 2006.

5. Kong, F.M.; Hayman, J.A.; Griffith, K.A.; et al. Final toxicity results of a radiation-dose escalation study in patients with non-small-cell lung cancer (NSCLC): Predictors for radiation pneumonitis and fibrosis. Int. J. Radiat. Oncol. Biol. Phys. 65:1075-86; 2006.

6. Allen, A.M.; Schofield, D.; Hacker, F.; et al. Restricted field IMRT dramatically enhances IMRT planning for mesothelioma. Int. J. Radiat. Oncol. Biol. Phys. 69:1587-92; 2007.

7. Allen, A.M.; Czerminska, M.; Jänne, P.A.; et al. Fatal pneumonitis associated with intensity-modulated radiation therapy for mesothelioma. Int. J. Radiat. Oncol. Biol. Phys. 65:640-5; 2006.

8. Sterzing, F.; Sroka-Perez, G.; Schubert, K.; et al. Evaluating target coverage and normal tissue sparing in the adjuvant radiotherapy of malignant pleural mesothelioma: Helical tomotherapy compared with step-and-shoot IMRT. Radiother. Oncol. 86:251-7; 2008.

9. Mehta, M.; Scrimger, R.; Mackie, R.; et al. A new approach to dose escalation in non-small cell lung cancer. Int. J. Radiat. Oncol. Biol. Phys. 49:23-33; 2001.

10. Lieskovsky, Y.C.; Koong, A.; Fisher, G.; et al. Phase I dose escalation study of CyberKnife stereotactic radiosurgery for liver malignancies. Int. J. Radiat. Oncol. Biol. Phys. 63:S283; 2005 . 
11. Mackie, T.R.; Holmes, T.; Swerdloff, S.; et al. Tomotherapy: A new concept for the delivery of dynamic conformal radiotherapy. Med. Phys. 20:1709-19; 1993.

12. Mackie, T.R.; Balog, J.; Ruchala, K.; et al. Tomotherapy. Semin. Radiat. Oncol. 9:108-17; 1999.

13. Bauman, G.B.; Yartsev, S.; Rodrigues, G.; et al. A prospective evaluation of helical tomotherapy. Int. J. Radiat. Oncol. Biol. Phys. 68:632-41; 2007.

14. Ramsey, C.R.; Langen, K.M.; Kupelian, P.A.; et al. A technique for adaptive image-guided helical tomotherapy for lung cancer. Int. J. Radiat. Oncol. Biol. Phys. 64:1237-44; 2006.

15. Ramsey, C.; Mahan, S.; Scaperoth, D.; et al. Image guided adaptive therapy for the treatment of lung cancer. Int. J. Radiat. Oncol. Biol. Phys. 60:S339; 2004.

16. Kupelian, P.A.; Ramsey, C.; Meeks, S.L.; et al. Serial megavoltage $\mathrm{CT}$ imaging during external beam radiotherapy for non-small-cell lung cancer: Observations on tumor regression during treatment. Int. J. Radiat. Oncol. Biol. Phys. 63:102-48; 2005.

17. Woodford, C.; Yartsev, S.; Dar, A.R.; et al. Adaptive radiotherapy planning on decreasing gross tumor volume as seen on megavoltage computed tomography images. Int. J. Radiat. Oncol. Biol. Phys. 69:1316-22; 2007.

18. Yartsev, S.; Dar, A.R.; Woodford, C.; et al. Initial experience in treating lung cancer with helical tomotherapy. Biomed. Imaging Interv. J. 3:e2; 2007

19. Sørensen, B.; Hao, J.; Overgaard, J.; et al. Influence of oxygen concentration and $\mathrm{pH}$ on expression of hypoxia induced genes. Radiother. Oncol. 76:187-93; 2005.
20. Rodrigues, G.; Lock, M.; D'Souza, D.; et al. Prediction of radiation pneumonitis by dose-volume histogram parameters in lung cancer - A systematic review. Radiother. Oncol. 71:127$38 ; 2004$.

21. Yartsev, S.; Chen, J.; Yu, E.; et al. Comparative planning evaluation of intensity-modulated radiotherapy techniques for complex lung cancer cases. Radiother. Oncol. 78:169-76; 2007.

22. Martel, M.K.; Ten Haken, R.K.; Hazuka, M.B.; et al. Estimation of tumor control probability parameters from 3-D dose distributions of non-small cell lung cancer patients. Lung Cancer. 24:31-7; 1999.

23. Saunders, M.; Dische, S.; Barrett, A.; et al. Continuous hyperfractionated accelerated radiotherapy (CHART) versus conventional radiotherapy in non-small cell lung cancer: A randomized multicenter trial. Radiother. Oncol. 52:137-48; 1999.

24. Werner-Wasik, M.; Xiao, Y.; Pequignot, E.; et al. Assessment of lung cancer response after nonoperative therapy: Tumor diameter, bidimensional product, and volume. A serial CT scan-based study. Int. J. Radiat. Oncol. Biol. Phys. 51:56-61; 2001.

25. Britton, K.R.; Starkschall, G.; Tucker, S.L.; et al. Assessment of gross tumor volume regression and motion changes during radiotherapy for non small-cell lung cancer as measured by four-dimensional computed tomography. Int. J. Radiat. Oncol. Biol. Phys. 68:1036-46; 2007.

26. Welsh, J.S.; Bradley, K.; Ruchala, K.J.; et al. Megavoltage computed tomography imaging: A potential tool to guide and improve the delivery of thoracic radiation therapy. Clin. Lung Cancer. 5:303-6; 2004. 